\title{
Multi Vessel Coronary Artery Disease Presenting as a False Negative Myocardial Perfusion Imaging and True Positive Exercise Tolerance Test: A Case of Balanced Ischemia
}

\author{
Abdul Baqi ${ }^{1}$, Intisar Ahmed ${ }^{2}$, Babar Nagher ${ }^{2}$ \\ 1. Cardiology, Aga Khan University Hospital, Karachi, PAK 2. Cardiology, Aga Khan University Hospital, Karachi, PAK
}

Corresponding author: Abdul Baqi,dr_abkhana@yahoo.com

\begin{abstract}
Non-invasive investigations play an important role in the early diagnosis of coronary artery disease. Although the stress test is an easily available investigation for the diagnosis of obstructive coronary artery disease, its results are affected by the pretest probability. Myocardial perfusion imaging (MPI) is one of the commonly performed non-invasive cardiac imaging. The common reasons for false-negative exercise MPI are reported to be inadequate exercise, a lower dose of radio-tracer, small area of perfusion defect, and ischemia caused by left circumflex or its branches. Balanced ischemia is one of the rare causes of falsenegative MPI. In this case report, we present a 73-year-old gentleman who presented with chest pain and shortness of breath. An exercise electrocardiogram (ECG) at five metabolic equivalents was positive for symptoms and electrocardiographic evidence of ischemia, but the myocardial perfusion image did not show ischemia. An invasive coronary angiogram was done due to high-risk exercise ECG, which revealed severe three-vessel coronary artery disease.
\end{abstract}

Although the false-negative myocardial perfusion scan in the presence of a positive exercise electrocardiogram is unusual, it should not be ignored. Positive exercise ECG with the reproduction of symptoms indicates a high probability of critical coronary artery disease, irrespective of perfusion defects.

Categories: Cardiac/Thoracic/Vascular Surgery, Cardiology, Internal Medicine

Keywords: balanced ischemia, myocardial perfusion imaging, exercise tolerance test

Review began 09/23/2020 Review ended 10/24/2020 Published 11/04/2020

\section{() Copyright 2020}

Baqi et al. This is an open access article distributed under the terms of the Creative Commons Attribution License CC-BY 4.0., which permits unrestricted use, distribution, and reproduction in any medium, provided the original author and source are credited.

\section{Introduction}

Coronary artery disease is the leading cause of mortality and morbidity in developed countries and its prevalence is growing exponentially in developing as well as under-developed countries [1]. Non-invasive investigations play an important role in the early diagnosis of coronary artery disease. Although stress test is the easily available and most commonly used investigation for the diagnosis of obstructive coronary artery disease, its results are affected by the pretest probability of the disease [2]. In patients with a low pretest probability of coronary artery disease, it has high rates of false-positive, and in those with high pretest probability, there are higher chances of false negatives. That is why it is recommended in patients with an intermediate pretest probability of coronary artery disease [3]. Myocardial perfusion imaging (MPI) is one of the commonly performed non-invasive cardiac imaging which has an additional role of detecting myocardial viability on top of establishing the diagnosis and prognosis of patients with coronary artery disease [4]. The radio-tracer used in myocardial perfusion imaging is distributed in the myocardium and a gamma camera detects the photons emitted by the radiotracer. The uptake of radio-tracer is dependent on perfusion, poorly perfused myocardium shows low uptake and well-perfused myocardium reveals high uptake of the radiotracer [5,6]. Although myocardial perfusion imaging has high sensitivity, a false-negative test is not uncommon. The common reasons for false-negative exercise MPI are reported to be inadequate exercise, a lower dose of radio-tracer, small area of perfusion defect, and ischemia caused by left circumflex or its branches [7]. Here we report a case of a patient having a false negative myocardial perfusion scan in the setting of multi-vessel coronary artery disease on invasive coronary angiogram.

\section{Case Presentation}

A 73-year-old gentleman presented to the cardiology clinic with chest pain on moderate exertion for the last two weeks, which was relieved at rest. He also complained of shortness of breath on moderate exertion with no history of resting symptoms and was asymptomatic at the time of presentation. He was recently diagnosed to have chronic lymphocytic leukemia (CLL) and had not been started on any treatment yet. He did not have any risk factors for coronary artery disease except advanced age and male gender.

On examination, he had a heart rate of 84 beats per minute, blood pressure of $135 / 75 \mathrm{mmHg}$, respiratory rate of 16 breaths per minute and an oxygen saturation of $98 \%$ while breathing in room air. Resting electrocardiogram (ECG) revealed sinus rhythm with no significant ischemic changes (Figure 1). 


\section{Cureus}

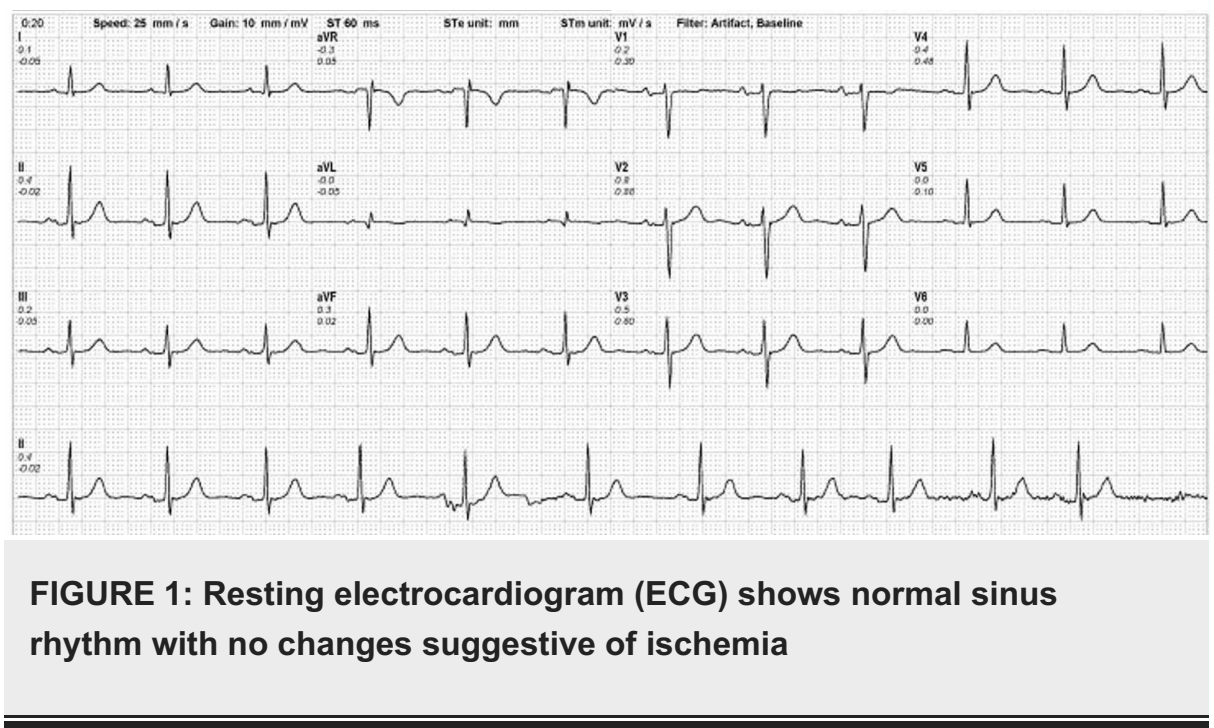

Other investigations revealed hemoglobin of $13.9 \mathrm{~g} / \mathrm{dl}$, total leukocyte count of 70.7x10E9/L, platelets count of 177 x10E9/L, serum creatinine of $0.7 \mathrm{mg} / \mathrm{dl}$, serum sodium of $143 \mathrm{mmol} / \mathrm{L}$, and hemoglobin A1c (HbA1c) was $5.8 \%$. His total serum cholesterol level was $159 \mathrm{mg} / \mathrm{dl}$, high-density lipoprotein (HDL) was $28 \mathrm{mg} / \mathrm{dl}$ and low-density lipoprotein (LDL) level was $92 \mathrm{mg} / \mathrm{dl}$. Trans-thoracic echocardiogram (TTE) showed an ejection fraction (EF) of 55\% with no segmental wall motion abnormality and there was no structural abnormality.

He was advised to get a stress myocardial perfusion imaging (MPI) for the assessment of ischemia. He underwent single-photon emission cardiac tomography (SPECT) with Technetium 99-m (Tc-99). The patient exercised for four minutes on the Bruce protocol and achieved $84 \%$ of the maximum predicted heart rate. Technetium-99 was injected at $84 \%$ of the age-predicted maximum heart rate. The test was stopped due to exercise limiting chest pain and dyspnea. ECG at peak stress showed $>1 \mathrm{~mm}$ horizontal ST depressions in leads II, III, aVF, and V3 to V5 along with ST elevation in lead aVR (Figure 2). ECG changes and symptoms were relieved at six minutes of recovery.

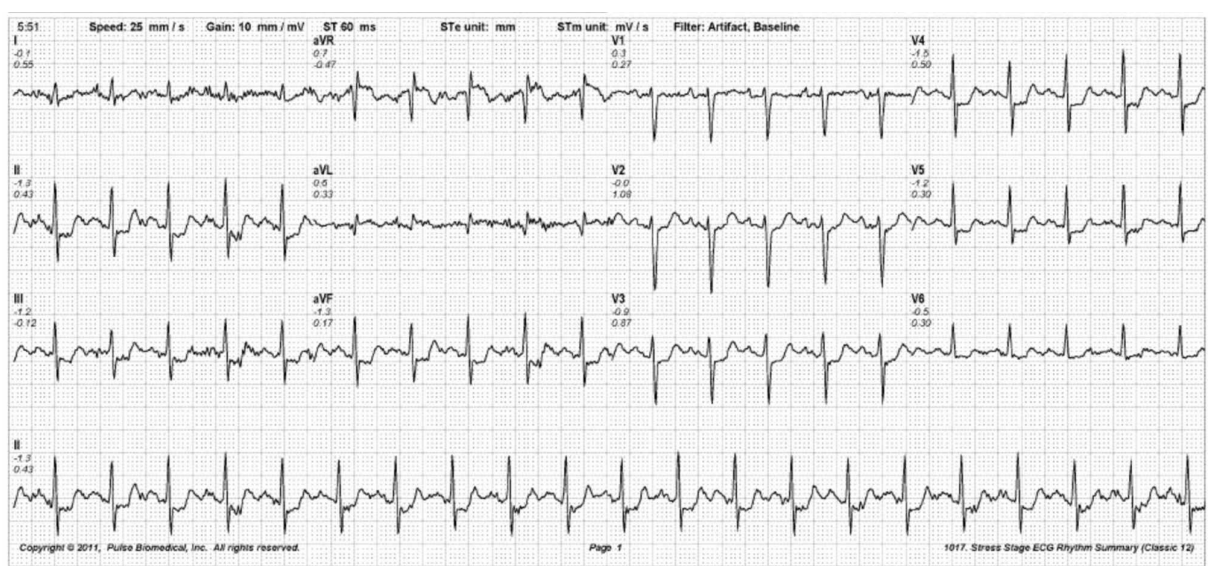

FIGURE 2: Electrocardiogram (ECG) at peak exercise showing more than $1 \mathrm{~mm}$ horizontal ST depression in precordial and inferior leads along with ST elevation in lead aVR.

Myocardial perfusion imaging (MPI) revealed a small-sized fixed perfusion defect of moderate-intensity in the basal inferior segment (Figure 3). 


\section{Cureus}

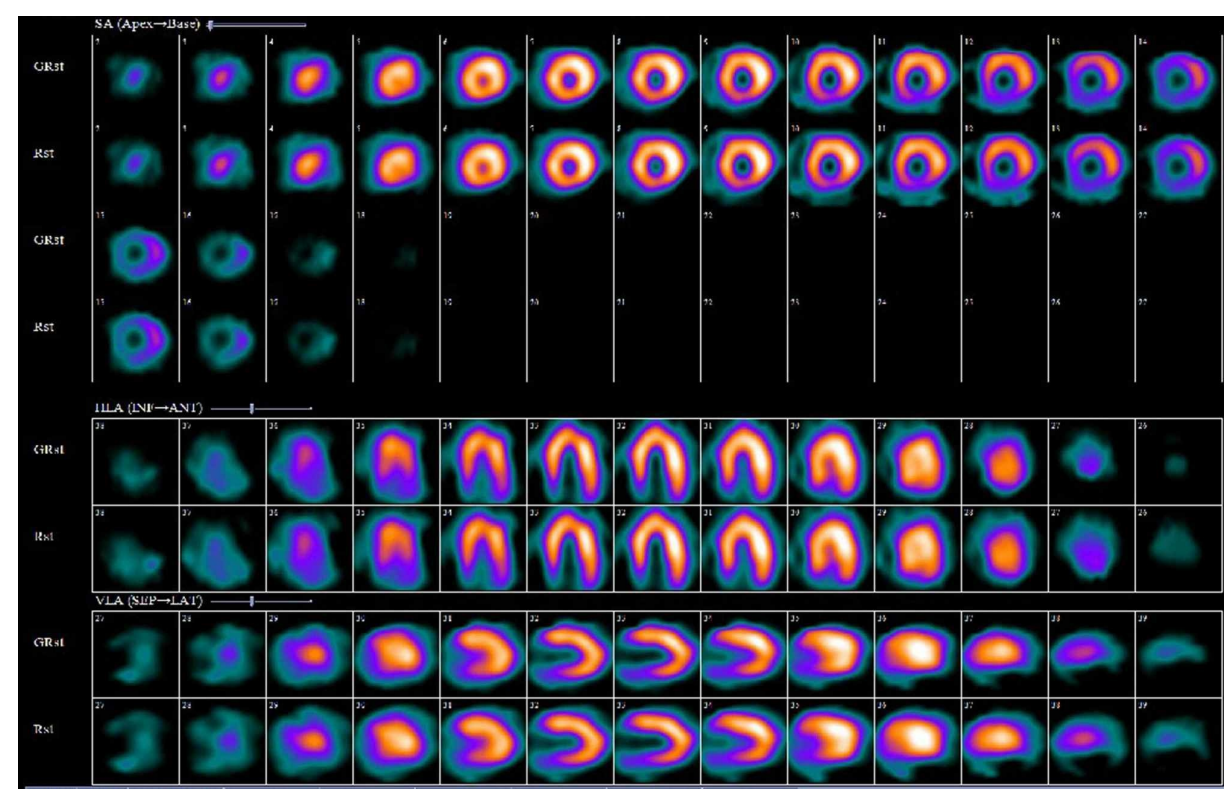

FIGURE 3: Myocardial perfusion scan showing small sized fixed perfusion defect involving the basal inferior segment.

Although MPI did not show any ischemia, exercise ECG was positive for symptoms and electrocardiographic evidence of myocardial ischemia. He was started on aspirin, rosuvastatin, bisoprolol, and then underwent an invasive coronary angiogram, which revealed multi-vessel coronary artery disease (Figures 4-๑).

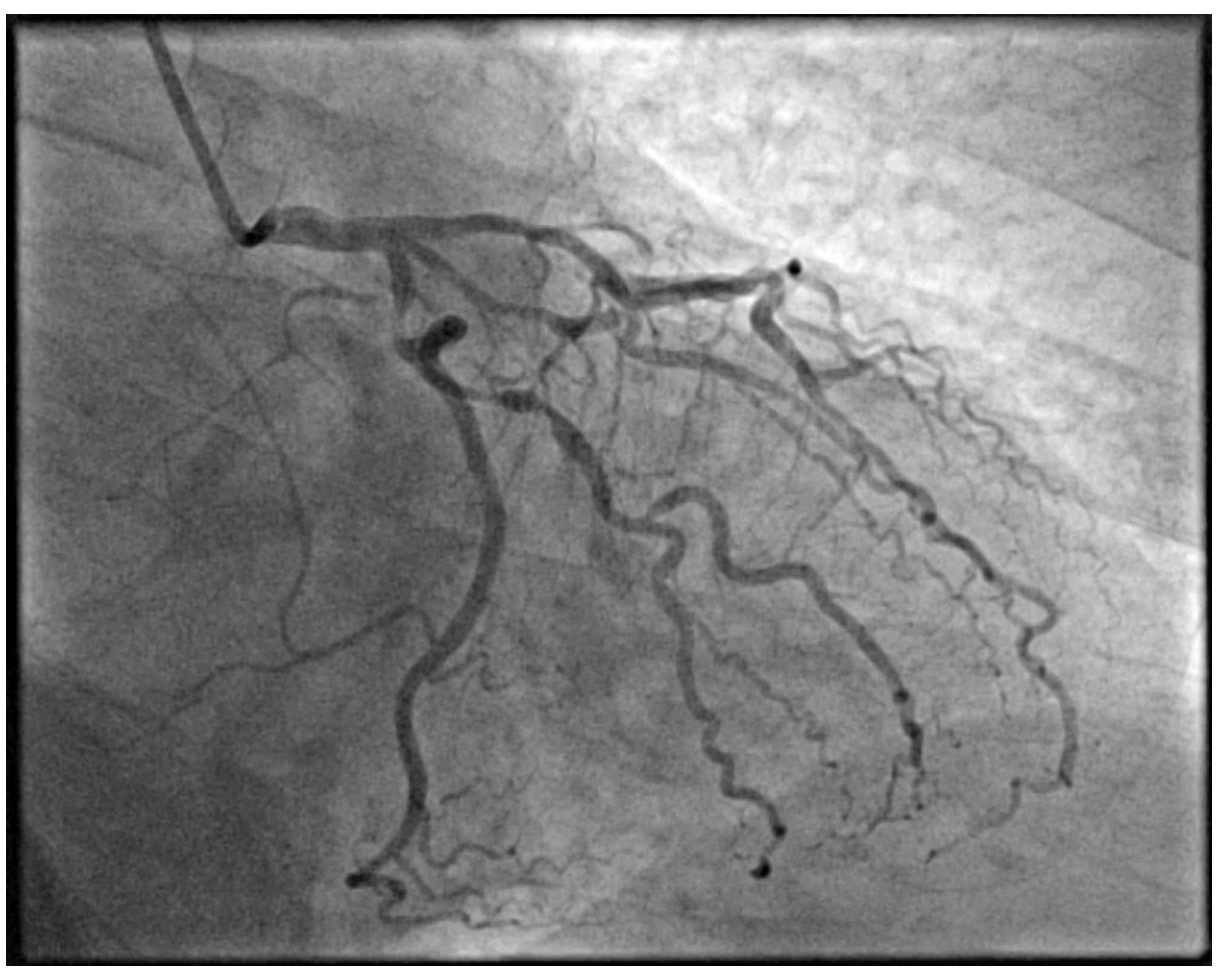

FIGURE 4: Caudal projection showing an obstructive lesion in the proximal segment of LCx, OM1, and OM2 branches.

LCx (Left circumflex), OM1 (Obtuse marginal 1), OM2 (Obtuse marginal 2) 


\section{Cureus}

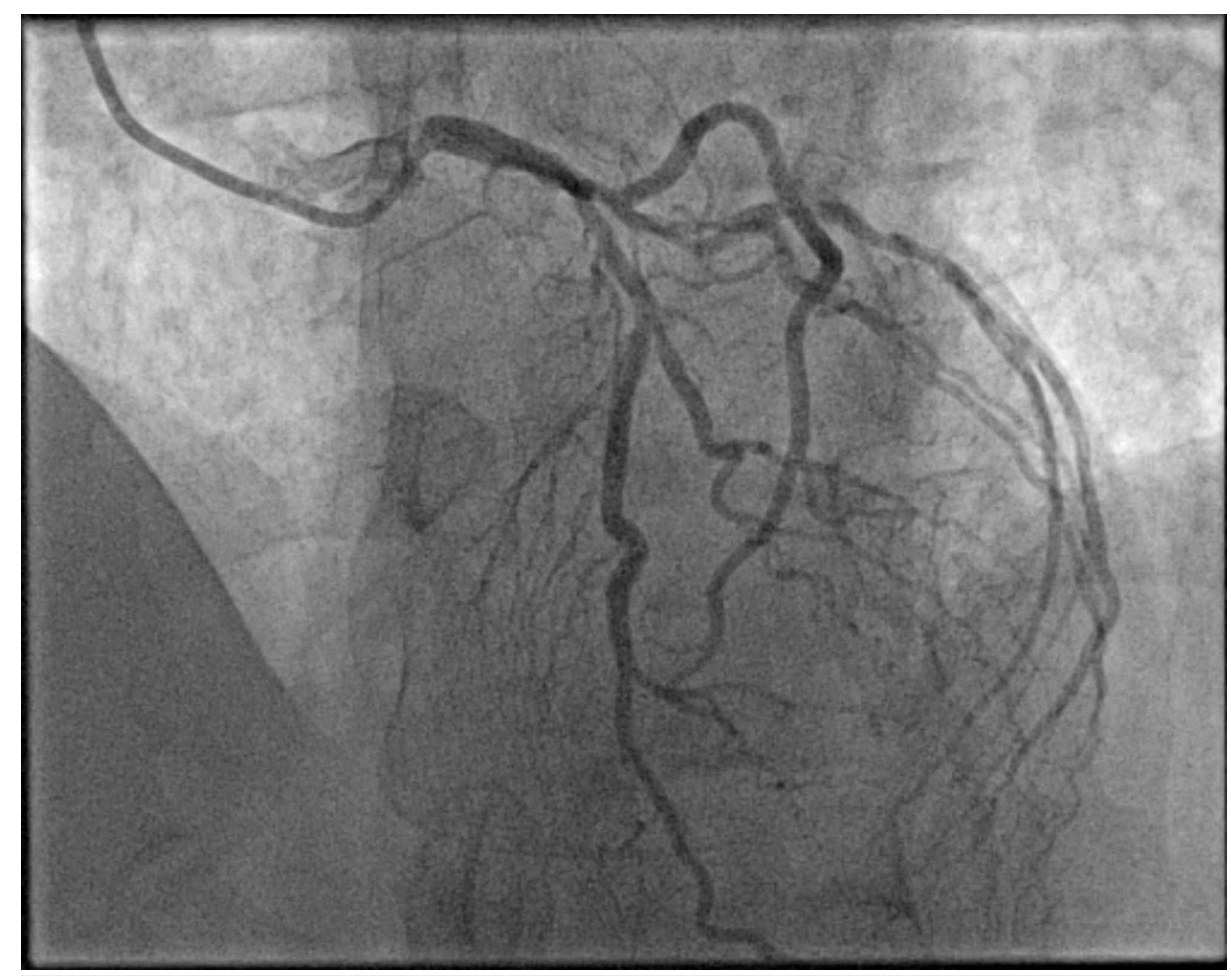

FIGURE 5: Cranial projection showing a significant obstructive lesion in the proximal and mid segments of the left anterior descending artery.

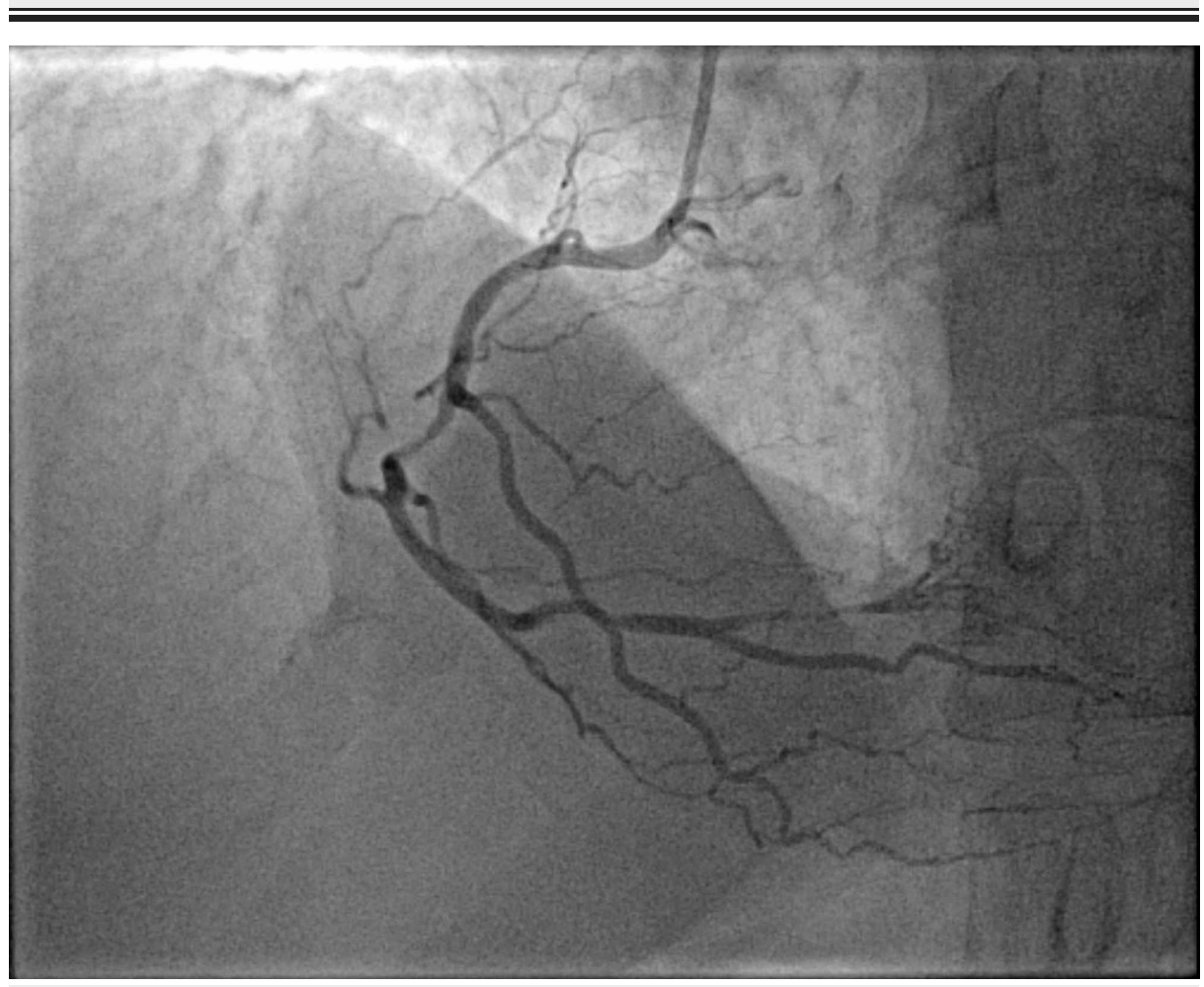

FIGURE 6: Cranial projection of the right coronary artery, showing obstructive lesions in the mid-segment of RCA and RPLV branch.

RPLV (right posterolateral ventricular), RCA (right coronary artery)

His current treatment was continued and long-acting isosorbide mononitrate added to his treatment regimen. After a detailed discussion with the patient and family members, he was referred to a cardiac 
surgeon for coronary artery bypass graft.

\section{Discussion}

In this case report, we described a patient with chest pain and shortness of breath, who was found to have electrocardiographic evidence of ischemia on exercise ECG but MPI did not show ischemia. Invasive coronary angiogram confirmed the diagnosis of severe multi-vessel coronary artery disease.

The exercise stress test is a major noninvasive diagnostic test for coronary artery disease, which is readily available and easy to interpret. A combination of exercise ECG and myocardial perfusion imaging has significantly increased the diagnostic accuracy of stress myocardial perfusion imaging [8].

Although SPECT MPI is reported as one of the most sensitive non-invasive tests for coronary artery disease and patients with negative MPI have been studied to have less than $1 \%$ incidence of major cardiovascular events for a year [9]. However, normal MPI has also been reported in patients with angiographically significant coronary artery disease. The possible causes of false-negative MPI in that situation may include branch vessel stenosis, left circumflex artery stenosis, inadequate exercise, and caffeine intake before MPI (for dipyridamole myocardial perfusion imaging) [10,11,12].

Balanced three-vessel coronary artery disease has been reported as one of the rare causes of false-negative myocardial perfusion scans [13]. Our patient had severe three vessels coronary artery disease and falsenegative MPI. But exercise electrocardiogram was positive for ischemia and his symptoms were reproduced on even low level of exercise, which makes a duke treadmill score of -11.5 , suggesting a high likelihood of coronary artery disease and high risk of future cardiovascular events [14].

The detection of ischemia on MPI is based on relative perfusion of different myocardial segments. The segment with maximum uptake is considered normal and other segments are compared with that. In the presence of balanced ischemia, although radiotracer uptake is reduced overall. It is equally distributed in this setting and is proposed to appear due to the unopposed apical ischemia thus considered diagnostic [15].

\section{Conclusions}

Although the false-negative myocardial perfusion scan in the presence of a positive exercise electrocardiogram is unusual, it should not be ignored. Positive exercise ECG with the reproduction of symptoms indicates a high probability of critical coronary artery disease, irrespective of perfusion defects.

\section{Additional Information \\ Disclosures}

Human subjects: Consent was obtained by all participants in this study. Conflicts of interest: In compliance with the ICMJE uniform disclosure form, all authors declare the following: Payment/services info: All authors have declared that no financial support was received from any organization for the submitted work. Financial relationships: All authors have declared that they have no financial relationships at present or within the previous three years with any organizations that might have an interest in the submitted work. Other relationships: All authors have declared that there are no other relationships or activities that could appear to have influenced the submitted work.

\section{Acknowledgements}

We acknowledge the help of the Nuclear Cardiology nursing staff of the Cardiology section Aga Khan University Hospital for helping us in the arrangement of logistics and during performing procedures. We also acknowledge the help of Cath Lab staff in helping with the required data.

\section{References}

1. Gaziano TA, Bitton A, Anand S, Abrahams-Gessel S, Murphy A: Growing epidemic of coronary heart disease in low-and middle-income countries. Curr Probl Cardiol. 2010, 35:72-115. 10.1016/i.cpcardiol.2009.10.002

2. Diamond GA, Forrester JS: Analysis of probability as an aid in the clinical diagnosis of coronary-artery disease. N Engl J Med. 1979, 14:1350-8. 10.1056/NEJM197906143002402

3. Wolk MJ, Bailey SR, Doherty JU, et al.: ACCF/AHA/ASE/ASNC/HFSA/HRS/SCAI/SCCT/SCMR/STS 2013 multimodality appropriate use criteria for the detection and risk assessment of stable ischemic heart disease: a report of the American College of Cardiology foundation appropriate use criteria task force. J Am Coll Cardiol. 2014, 63:380-406. 10.1016/j.jacc.2013.11.009

4. Fathala A: Myocardial perfusion scintigraphy: techniques, interpretation, indications, and reporting . Ann Saudi Med. 2011, 31:625-34. 10.4103/0256-4947.87101

5. Kotler TS, Diamond GA: Exercise thallium-201 scintigraphy in the diagnosis and prognosis of coronary artery disease. Ann Intern Med. 1990, 113:684-702. 10.7326/0003-4819-113-9-684

6. Beller GA, Watson DD: Physiological basis of myocardial perfusion imaging with the technetium 99m agents. Semin Nucl Med. 1991, 21:173-181). WB. 10.1016/s0001-2998(05)80038-8

7. Yokota S, Mouden M, Ottervanger JP: High-risk coronary artery disease, but normal myocardial perfusion: a 


\section{Cureus}

matter of concern?. J Nucl Cardiol. 2016, 23:542-545. 10.1007/s12350-015-0167-7

8. Nagashima JI, Yamada H, Ohkawa SI, et al.: Evaluation of thallium-201 myocardial emission computed tomography based on a comparison with postmortem findings [Article in Japanese]. Radioisotopes. 1986, 35:631-5. 10.3769/radioisotopes.35.12_631

9. Wackers FJ, Russo DJ, Russo D, Clements JP: Prognostic significance of normal quantitative planar thallium-201 stress scintigraphy in patients with chest pain. J Am Coll Cardiol. 1985, 6:27-30. 10.1016/s0735-1097(85)80247-3

10. Osbakken MD, Okada RD, Boucher CA, Strauss HW, Pohost GM: Comparison of exercise perfusion and ventricular function imaging: an analysis of factors affecting the diagnostic accuracy of each technique. J Am Coll Cardiol. 1984, 3:272-83. 10.1016/s0735-1097(84)80010-8

11. Rigo P, Bailey IK, Griffith LS, Pitt B, Wagner HN, Becker LC: Stress thallium-201 myocardial scintigraphy for the detection of individual coronary arterial lesions in patients with and without previous myocardial infarction. Am J Cardiol. 1981, 48:209-16. 10.1016/S0002-9149(05)80002-5

12. Smits P, Corstens FH, Aengevaeren WR, Wackers FJ, Thien T: False-negative dipyridamole-thallium-201 myocardial imaging after caffeine infusion. J Nucl Med. 1991, 32:1538-41.

13. Aarnoudse WH, Botman KJ, Pijls NH: False-negative myocardial scintigraphy in balanced three-vessel disease, revealed by coronary pressure measurement. Int J Cardiovasc Intervent. 2003, 5:67-71. $10.1080 / 14628840310003244$

14. Pryor DB, Shaw L, Harrell Jr FE, et al.: Estimating the likelihood of severe coronary artery disease . Am J Med. 1991, 90:553-62.

15. Madias JE, Knez P, Win MT: True-positive exercise electrocardiogram/false-negative thallium-201 scintigram: a proposal of a mechanism for the paradox. Clin Cardiol. 2000, 23:625-9. 10.1002/clc.4960230815 